\title{
Development of Mammalian Cell Lines with lac Operator-Tagged Chromosomes
}

\author{
Yuri G. Strukov and Andrew S. Belmont
}

This protocol was adapted from "Development of Mammalian Cell Lines with lac Operator-Tagged Chromosomes," Chapter 25, in Live Cell Imaging (eds. Goldman and Spector). Cold Spring Harbor Laboratory Press, Cold Spring Harbor, NY, USA, 2005.

\section{INTRODUCTION}

The discovery and use of fluorescent proteins to label chromosomal proteins has yielded basic structural information as well as insights into dynamics that were previously inaccessible. This protocol describes a method for tagging specific chromosome sites using the lac operator/repressor system, wherein direct repeats of bacterial operator sequences are coupled with green fluorescent protein (GFP)-tagged proteins that recognize these sequences. Direct lac operator repeats are generated by directional cloning. Although the use of direct repeats, as opposed to inverted repeats, reduces recombination within the bacterial host at high copy number, even the direct repeats are unstable, requiring the use of special bacterial hosts and low-copy-number plasmids for cloning. The introduction of the lac operator repeats into eukaryotic cells uses traditional transformation methods. Techniques for the isolation of stable cell clones with varying transgene copy numbers are described in the protocol, as are several methods for visual screening of large numbers of stable cell clones to isolate rare clones containing labeled chromosomal regions with desired features.

\section{RELATED INFORMATION}

In principle, chromosome tagging can be accomplished through the use of any repetitive sequence to which a specific protein binds. The first application of this approach used the bacterial lac operator and Lac repressor combination. The lac operator/Lac repressor-labeling system has been successfully adapted for tagging chromatin in live bacteria, yeast, and mammalian tissue culture cells (Fig. 1), as well as in multicellular organisms including Caenorhabditis elegans, Drosophila, and Arabidopsis. Subsequent approaches have used tet operator or glucocorticoid receptor element repeats. Recently, the lac operator system has been used in combination with the bacteriophage viral replicase translational operator and several color variants of GFP to label DNA, RNA, and protein in living cells.

\section{MATERIALS}

CAUTIONS AND RECIPES: Please see Appendices for appropriate handling of materials marked with $<!>$, and recipes for reagents marked with $<\mathbf{R}>$.

\section{Reagents}

Agarose gel (0.7\%) 
$<$ !>Ampicillin (add at $100 \mu \mathrm{g} / \mathrm{mL}$ to both LB medium and LB agar plates)

Bacteria (e.g., Stbl2 competent cells; Invitrogen/GIBCO)

$<\mathrm{R}><$ ! $>\mathrm{CaCl}_{2}(250 \mathrm{mM})$

Cells for transfection: CHO DG44

DNA preparation kits (e.g., QIAGEN miniprep and maxiprep kits)

Ethanol (70\%; ice-cold 100\%)

Fetal bovine serum (FBS), defined and dialyzed (HyClone Lab)

$<$ !>Fluorescein-labeled methotrexate (FMTX) (Molecular Probes)

Glycine $(100 \mu \mathrm{M})$

Hypoxanthine $(30 \mu \mathrm{M})$

$<\mathrm{R}>\mathrm{LB}$ medium (liquid and agar plates)

Media for cells used for transfection

F-12 (HAM) medium (Invitrogen/GIBCO) supplemented with 10\% defined FBS: for growth of DG44 cells

F-12 medium, lacking thymidine and hypoxanthine, with 10\% dialyzed FBS: for selection and further passaging of stably transfected cells

$<\mathbf{R}>$ Phosphate-buffered saline, calcium- and magnesium-free (CMF-PBS)

Restriction enzymes Sall and Xhol

<!> Sodium acetate (3 M, pH 5.5)

TE buffer (optional; see Step 4.vii)

Thymine $(30 \mu \mathrm{M})$

$<$ R $>$ TR buffer

$<$ !>Trypsin

Vector (e.g., psv2-DHFR-8.32) and appropriate insert (e.g., 256-copy lac operator repeat) (see Steps 1 and 2)

\section{Equipment}

\section{$<$ ! Dry ice}

Equipment for agarose gel electrophoresis

Filter paper

Flasks (25- $\mathrm{cm}^{2}$ tissue culture; $\left.75-\mathrm{cm}^{2}\right)$

Fluorescence-activated cell sorter

Forceps (sharp-tipped)

Incubator preset to $30^{\circ} \mathrm{C}$

Inoculation loops

Marker (permanent)

Microcentrifuge

Microcentrifuge tubes $(1.5 \mathrm{~mL})$

Micropipette tips (sterile $200-\mu \mathrm{L})(1 \mathrm{~mL})$ (optional; see Step 8.viii)

Microscope

Petri dishes (150-mm)

Pipette

Plastic hose (optional; see Step 8.viii)

Plates for growing bacterial cultures

Plates (96-well)

Shaking platform

Tubes for transfection

\section{METHOD}

\section{Preparing Vector DNA Containing Large Direct Repeats}

1. Clone the lac operator repeat into a vector. 


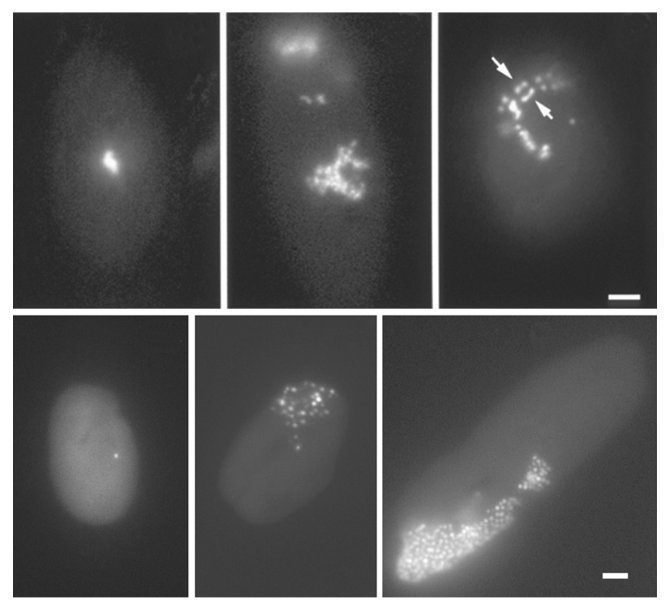

FIGURE 1. Examples of different stable cell lines with lac operator-tagged chromosome regions visualized by GFP-Lac repressor in vivo expression. (Top) Three different gene-amplified cell lines, derived from multicopy plasmid insertions, each with different characteristic conformations of the lac operator-tagged chromosome region. (Top, left) A03 cells with a heterochromatic-like amplified chromosome region. A 90-Mb region forms an $\sim 1-\mu \mathrm{m}$ diameter spot during most of interphase, not much larger than its $\sim 1.3-\mu \mathrm{m}$ metaphase chromosome length. (Top, middle) PDC cell line in which the amplified chromosome region appears as coiled, large-scale chromatin fibers by light and electron microscopy. (Top, right) $\mathrm{Bb}$ cell line in which extended large-scale chromatin fibers are observed. In this example, what appear to be two sister chromatids are shown. (Bottom) Different stages of gene amplification. (Bottom, left) EP1-4 cell line containing a single-copy insertion of plasmid transgene containing lac operator direct repeats. (Bottom, middle and bottom, right) Two different stages of gene amplification using methotrexate selection. Chromosome regions consisting of clusters of individual dots are observed. These increase in number with increased amplification between bottom, middle, and bottom, right. Bars (top), $1 \mu \mathrm{m}$; (bottom), $2 \mu \mathrm{m}$.

This protocol is based on preparations from our laboratory using the pSV2-DHFR-8.32 plasmid containing a 256-copy lac operator repeat.

i. Use Stbl2 competent cells grown at $30^{\circ} \mathrm{C}$ to help maintain full-length lac operator repeats.

ii. Clone the lac operator repeats into low-copy-number vectors (e.g., psv2-DHFR-8.32).

Having too many copies in a cell increases the possibility of recombination.

2. Purify vector DNA as follows:

The purification of vector DNA should take no more than $3 d$ to complete ( $4 d$ if the optional second minipreps are performed in Step2.viii). Recombination may occur if plates or cultures from Step 1 are allowed to stand at $4^{\circ} \mathrm{C}$; thus it is important to complete this procedure without delay. However, the plasmid DNA, once prepared from the cells, is stable. Because low-copy-number plasmids are used, expect low yields at every purification step.

i. Streak bacteria from a stab culture onto an LB plate containing ampicillin $(100 \mu \mathrm{g} / \mathrm{mL})$. Store the stab culture in the dark at room temperature.

ii. Incubate the inoculated LB plate for $24 \mathrm{~h}$ at $30^{\circ} \mathrm{C}$.

Even after this length of time, the colonies will be small.

iii. Inoculate at least $103-\mathrm{mL}$ cultures of LB containing ampicillin $(100 \mu \mathrm{g} / \mathrm{mL})$ with well-isolated colonies. Incubate the cultures on a shaker overnight at $30^{\circ} \mathrm{C}$.

iv. Prepare DNA using a miniprep procedure (e.g., QIAGEN) from $1.5 \mathrm{~mL}$ of each culture. Store the remainder of each culture at $4^{\circ} \mathrm{C}$.

v. Digest aliquots of the DNA with Sall and Xhol. Separate the DNA fragments on an agarose gel $(0.7 \%)$.

At this step, it is critical to be very discerning. Any bands present other than those expected could indicate recombination of the plasmid (in the case of the PSV2-DHFR-8.32 plasmid containing a 256-copy lac operator repeat, expect 10-kb and 5-kb fragments). 
vi. Prepare and freeze a glycerol stock, or prepare a stab culture of each positive clone (from the stored cultures in Step 2.iv).

vii. Inoculate $500 \mathrm{~mL}$ of $\mathrm{LB}$ containing ampicillin $(100 \mu \mathrm{g} / \mathrm{mL})$ with the remaining culture from one of the positive clones. Incubate the culture overnight at $30^{\circ} \mathrm{C}$ with shaking.

It may be preferable to prepare two $500-\mathrm{mL}$ cultures, each from a different positive clone, in the event that one clone is lost during large-scale growth.

viii.(Optional) Before proceeding with the maxiprep DNA isolation, isolate DNA from an aliquot of the 500-mL culture by repeating Steps 2.iv-2.v. While preparing the miniprep DNA, centrifuge the 500-mL culture. Discard the supernatant, and freeze the bacterial pellet until ready to use. Use positive cultures for the following step:

ix. Isolate DNA from the 500-mL culture using a maxiprep procedure. If using QIAGEN maxipreps with Qiafilters, double the volume of P1, P2, and P3 added to each pellet, and then split the prep into two Qiafilters. This prevents the Qiafilter from clogging.

The yield of DNA will be low. A good yield is $\sim 125 \mu \mathrm{g}$ of DNA per 500-mL culture.

x. Digest aliquots of the DNA with Sall and Xhol to confirm that only DNA fragments of the expected size are present.

\section{Making Stable Cell Lines Containing lac Operator Repeats}

3. Grow cells for transfection for $\sim 1 \mathrm{wk}$.

CHO DG44 cells are derivatives of CHO K1 cells and are doubly deleted for the DHFR locus. The product of the DHFR gene is necessary for intermediary metabolism of thymidylates and purines. An imbalance of nucleotides in cells may be the cause of both insert instability due to gene amplification and an abnormal cell cycle due to interference with replication timing.

4. Purify plasmid DNA for transfection as follows:

i. Transfer $300 \mu \mathrm{L}$ of vector DNA solution $(\sim 100 \mu \mathrm{g} / \mathrm{mL})$ into a 1.5-mL microcentrifuge tube.

ii. Add $33 \mu \mathrm{L}$ of sodium acetate ( $3 \mathrm{M}, \mathrm{pH} 5.5)$ to the DNA, and thoroughly mix the solution with a pipette.

iii. Add $660 \mu \mathrm{L}$ of ice-cold $100 \%$ ethanol to the tube, and mix the contents by inverting the tube several times. Let the tube stand on dry ice for $5 \mathrm{~min}$.

At this step, white fibers of DNA precipitate should be visible.

iv. Centrifuge the reaction in a microcentrifuge at $14,000 \mathrm{rpm}$ for $15 \mathrm{~min}$ at $4^{\circ} \mathrm{C}$. For convenience, label the location of the pellet inside the tube with a permanent marker.

v. Remove the supernatant, and wash the pellet with $1 \mathrm{~mL}$ of $70 \%$ ethanol at $4{ }^{\circ} \mathrm{C}$ by inverting the tube several times. Centrifuge the tube in a microcentrifuge at 14,000 rpm for 10 $\min$ at $4^{\circ} \mathrm{C}$.

Carry out all subsequent steps in a sterile environment.

vi. Remove the supernatant, and immerse the entire open tube in $70 \%$ ethanol for $10 \mathrm{sec}$. Remove the supernatant. Air-dry the DNA pellet for 10-15 min.

vii. Dissolve the dried DNA in $200 \mu \mathrm{L}$ of sterile $\mathrm{H}_{2} \mathrm{O}$ or TE buffer.

viii.Aliquot DNA dissolved in $\mathrm{H}_{2} \mathrm{O}$ and freeze at $-20^{\circ} \mathrm{C}$ for short-term storage. DNA dissolved in TE buffer is stable for at least several weeks at $4^{\circ} \mathrm{C}$.

ix. Remove an aliquot of the DNA, and estimate the concentration and purity of the DNA.

5. Transform cells using calcium-phosphate-precipitated DNA.

Use only chemical transformation with competent cells, not electroporation, because increased recombination following electroporation has been observed. The efficiency of transfection using calcium-phosphateprecipitated DNA strongly depends on the growth state of recipient cells, concentration of calcium and phosphates, concentration of DNA, pH, temperature, and duration of the precipitation reaction. It is advisable to prepare several buffers with slightly different $\mathrm{pH}$ values (see Step5.iii below).

i. Plate CHO DG44 cells in three $75-\mathrm{cm}^{2}$ flasks the day before transfection. 
ii. Replace the medium in the flasks with fresh medium $4 \mathrm{~h}$ before transfection. Cells should be $50 \%-80 \%$ confluent and be in the log phase of growth for efficient transfection.

iii. Prepare three reaction tubes. In each one, dilute $7 \mu \mathrm{g}$ of supercoiled plasmid DNA (from Step 4.vii) in $0.5 \mathrm{~mL}$ of $\mathrm{CaCl}_{2}(250 \mathrm{mM})$, mixed with $0.5 \mathrm{~mL}$ of TR buffer, varying only in its $\mathrm{pH}$ value $(6.95,7.05$, or 7.10$)$. Incubate the reactions for $30 \mathrm{~min}$ at room temperature.

iv. Add each DNA mixture dropwise to a flask of cells (from Step 5.ii). Incubate the flasks for $24 \mathrm{~h}$.

v. Replace the transfection medium with F-12 (HAM) medium supplemented with $10 \%$ defined FBS, and incubate the cells for an additional $24 \mathrm{~h}$.

vi. Replace the medium with selective medium (F-12 medium lacking thymidine and hypoxanthine, but containing 10\% dialyzed FBS). Stable transformants are obtained after 2 wk of selection.

\section{Enrichment of Stable Cells with Large-Copy-Number Inserts Using Fluorescence-Activated Cell Sorting}

This procedure describes the selection of CHO cells expressing high amounts of exogenous DHFR from a pool of stably transformed cells using cell sorting, a strategy to enrich for cells harboring large chromosome insertions of the DHFRcontaining transgene. Selection is based on binding a fluorescent cell-permeable DHFR inhibitor to the pool of cellular DHFR. Cells with high fluorescence show a high total expression of DHFR, which correlates roughly with copy number (as well as chromosome position effects).

6. Select and sort the cells as follows:

i. Grow cells in $25-\mathrm{cm}^{2}$ tissue culture flasks until they are in log phase.

ii. Replace the growth medium with fresh F-12 (HAM) medium supplemented with FBS (10\%) and FMTX $(20 \mu \mathrm{M})$. Add glycine $(100 \mu \mathrm{M})$, hypoxanthine $(30 \mu \mathrm{M})$, and thymine $(30 \mu \mathrm{M})$ to the medium to relieve the toxic effects of FMTX. Incubate the cells for $8 \mathrm{~h}$.

iii. Harvest cells by trypsinization, and keep them in sterile PBS on ice.

Because FMTX is retained in cells at concentrations high enough to cause damage, cells are viable in PBS on ice only for several hours.

iv. Sort the cells into flasks or 96-well plates.

v. Grow enough cells for freezing, and freeze them after screening.

7. Prepare a large number of clones for screening or subcloning as follows:

i. Plate cells produced in Step 6 onto a Petri dish at a low density, ideally, 20-100 cells per $150-\mathrm{mm}$ dish. To minimize cross-contamination among neighboring colonies, make sure that the density of plating is low enough and that the colonies are spread out. Use several Petri dishes, titrating the cell concentration by factors of 2-3 between dishes, to ensure optimal cell density.

The action of the trypsin (Step 6.iii) must have progressed enough to ensure the absence of cell clumping and the predominance of single cells in suspension. This is aided by mechanical agitation or by vigorous pipetting of the culture.

ii. Return the cells to the incubator, and if possible, avoid moving the dishes (particularly after the first few days) for $\sim 10 \mathrm{~d}$ to allow good-size colonies to form.

Movement of the dishes will cause cells to dislodge and form satellite colonies and/or a lawn of dispersed cells, increasing cross-contamination.

iii. Allow the cells to grow until the colonies are well-defined and visible by eye while avoiding overgrowth to minimize colony cross-contamination. Mark individual isolated colonies on the bottom of the plate with a permanent marker. View the colonies under low magnification to check for the presence of undesirable smaller colonies growing in the vicinity of any colony of interest.

8. If visual screening of the clones is unnecessary, then pick and harvest a large number of colonies from the 150-mm-diameter Petri dish (from Step 7), using one of the subcloning methods 
described below. If live visual screening is desired, refer to the Discussion regarding subcloning stable transformants.

\section{Trypsin Method for Picking Colonies}

i. Cut filter paper into small squares, each with an area of $\sim 4 \mathrm{~mm}^{2}$. Use sharp-tipped forceps to place the squares together in a glass Petri dish. Cover them and sterilize by autoclaving.

ii. Aspirate the medium from the Petri dish containing the marked colonies (from Step 7.iii above).

iii. Wash the cells with sterile calcium- and magnesium-free PBS (CMF-PBS), and remove excess liquid.

iv. Dispense trypsin solution into a small sterile Petri dish. Using the sterile forceps, dip a sterile filter paper (from Step 8.i) in trypsin, blot it to remove excess trypsin, and place it over a colony.

v. Repeat Step 8.iv for each of the colonies. Return the Petri dish containing the cells to the incubator for $\sim 5 \mathrm{~min}$.

vi. Transfer each filter paper into a separate well of a 96 -well microtiter plate containing $0.1 \mathrm{~mL}$ of tissue culture medium per well. If visual screening will be performed, use a glass-bottom 96-well plate. Duplicate plating can be accomplished by dipping the filter paper into one well to dislodge some cells, and then dropping the filter paper into a well of a second 96-well plate.

The efficiency of colony duplication by this method is close to 100\%. Do not leave the cells without buffer for more than 15-20 min or they will dry out. Also, CMF-PBS may induce rounding of cells and/or detachment after prolonged treatment (see Steps 8.x and 8.xi).

vii. Grow the collected cells in the 96-well plates for 1-2 d.

viii. Remove the filter paper pieces from the wells, taking care to avoid cross-contamination. One method for doing this is to attach a plastic hose to a vacuum system with an in-line trap. Insert the larger end of a 1-mL micropipette tip into the hose. Cut the other end of the tip to accommodate a sterile $200-\mu \mathrm{L}$ micropipette tip. Next, insert the $200-\mu \mathrm{L}$ tip into the bottom of a well. The vacuum will cause the filter paper to adhere to the tip. Withdraw the tip, and discard both the filter paper and the $200-\mu \mathrm{L}$ pipette tip. Finally, repeat with the remaining wells, using a fresh sterile 200$\mu \mathrm{L}$ tip each time.

ix. Continue to grow the cells until their numbers are sufficient for screening. Screen the cells from each well, and freeze the positive clones. Examine the positive clones by microscopy for the biological activity of interest.

\section{Micropipette Tip Method for Picking Colonies}

This method works well with more densely packed colonies, and it is faster than the trypsin method (Steps 8.i-8.ix). A few hundred colonies can be easily transferred within a couple of hours. Once this method is optimized, efficiency is near $100 \%$, although the number of cells transferred per colony into the well may be somewhat lower.

x. Mark the colonies that are less likely to be cross-contaminated with cells from other colonies. Rinse the cells twice with CMF-PBS (10 mL per 150-mm dish). Add a small amount of CMF-PBS ( 4-6 $\mathrm{mL}$ ), just sufficient to cover the surface of the dish, and return the dish to the incubator.

xi. After $\sim 5 \mathrm{~min}$, look at the cells under a microscope to see if cells in colonies have rounded. If not, incubate further. Once the cells in the colony have rounded, but are still attached, they can be picked.

xii. Use a permanent marker to circle small groups of colonies on the bottom of the dish. This helps to keep track of which colonies have already been harvested.

xiii. Using a fresh $200-\mu \mathrm{L}$ pipette tip per colony, scrape two to three times over the colony while aspirating $10 \mu \mathrm{L}$ into the pipette tip. Insert the pipette tip into the well of a 96-well plate, pipetting medium from the well several times into the tip to wash the cells off into the well.

xiv. Grow cells until their numbers are sufficient for screening. Screen the cells from each well, and freeze the positive clones. Examine the positive clones by microscopy for the biological activity of interest. 


\section{DISCUSSION}

In general, screening of clones uses either a visual, microscope-based screen (if possible) or a molecular or biochemical screen. In some cases, both might be combined, either as two sequential screens or one parallel screen. An example from our work is a screen of a set of 96 subclones transfected with a nonfluorescent VP16 transcriptional activator fusion protein. A visual immunofluorescence assay was used to test directly for expression of the activator, and a transient transfection expression assay was run to test the capability of each clone to activate a luciferase reporter gene. The intersection of these two screens yielded a clone that induced chromosome repositioning after induction of VP16 targeting.

A starting point for subcloning is to determine roughly what fraction of stable transformants show the desired properties. If this fraction is large, then we typically carry out the initial transformation in a single flask, followed by subsequent subcloning of the mixed stable transformant pool by serial dilution cloning into 96-well plates. A range of dilutions should be used to ensure success. The advantage of serial dilution is that the investigator can more easily verify whether the cells in a given well actually arise from a single clone. If the fraction of desired cells is small (a few percent or less), and if a visual screen is possible, we prepare a single coverslip from the mixed pool of stable transformants and obtain an initial estimate of exactly how many clones need to be screened. One approach is to initially subclone in pools of clones (e.g., five to 10 per well). In theory, this should allow cloning of even rare transformants through several stages of subcloning. Often, however, the desired subclones are rare because of a selective bias against growth of these clones. In this case, if the pool size is too great, the percentage of desired cells can drop in the culture prior to the next stage of subcloning, producing diminishing returns. A better approach, where possible, is cell sorting with flow cytometry to enrich the cell population with cells containing the desired expression levels (see Step 6 above). When large numbers of clones are required, and if visual screening is unnecessary, we simply pick large numbers of colonies from a 150-mm-diameter Petri dish, using either small, trypsin-soaked pieces of filter paper (Steps 8.i-8.ix above) or micropipette tips (Steps 8.x-8.xiv above). These are used to deposit clonal cells into 96-well plates. Both methods are very rapid but have a high risk of clone contamination, due to the use of a single Petri dish. Any clones selected, based on a secondary screen, must go through an additional round of clone "purification" using serial dilution.

Live visual screening is a little more complicated. An easy, low-cost method is to cover a large Petri dish (i.e., $60-\mathrm{mm}$ diameter) with a layer of small, round coverslips (12-mm diameter). Cells are plated at low density such that one to five colonies grow per coverslip. Under sterile conditions, the coverslips are transferred to a Petri dish containing a hole over which a large glass coverslip is attached with vacuum grease and transported to the microscope for visual screening. Each colony on a given coverslip is screened. If a positive clone is found, then the coverslip is recovered under sterile conditions, and the region of the coverslip containing the desired colony is broken off with a diamond pen and passaged. A more convenient, but more expensive, method is to pick up a large number of colonies, as described above, and place them into a 96-well, glass-covered microtiter dish (Whatman, Polyfiltronics). These dishes are available with number 1-1/2 thickness coverslips suitable for highresolution imaging. After the cells in each well grow to a sufficient density, the 96-well plate can be directly screened using an inverted microscope. These plates, however, are expensive (about $\$ 25$ per plate at the time of publication) and in high demand; thus they may be back-ordered for 3-6 mo at a time. Also, significant variability exists between batches of plates in their ability to support cloning at high efficiency. This appears to be related to contamination of the plates with residual glue. Our experience is that many lots of these plates support cell growth at high density but not at the limiting dilutions required for cloning.

It is important to formulate a general approach to determine whether the biological phenomenon under study can be successfully recapitulated during biological imaging. In our experience, phototoxicity remains a serious problem. This is especially true for experiments that exploit the labeling of small, selected chromosome regions, where high magnification, low signal intensity, and correct focus require significant photoexposure. Different biological phenomena show very different thresholds for phototoxicity. Whereas chromosome decondensation after mitosis or rapid constrained chromosome motions appear to be relatively photoinsensitive, long-range chromosome movements induced by targeting of VP16 are extremely photosensitive, and VP16-induced large-scale chromatin decondensation is somewhere in between. Thus, most live-cell imaging studies should begin with a careful statistical survey using a fixed-cell approach. The goal is to define a biological phenomenon using standard cytological approaches and generate statistics that can be used to monitor reproducibility in a corresponding live-cell imaging experiment. If the statistics are not reproducible, then there is a 
problem with either the conditions used to grow the cells on the microscope stage or phototoxicity. In the latter case, the statistical approach can be used to determine the maximum photoexposure permitted that still allows "the biology" to proceed normally. If the phenomenon cannot be studied with fixed samples, for example, characterization of chromatin dynamics, then different approaches must be taken. At a minimum, we would like to demonstrate that the phenomenon being measured does not vary as a function of light exposure or time the cells spend being grown and imaged on the microscope stage, assuming that no time dependence is expected.

\section{ACKNOWLEDGMENTS}

This work was supported by National Institutes of Health grants GM-42516 and GM-58460 to A.S.B. 


\title{
Development of Mammalian Cell Lines with lac Operator-Tagged Chromosomes
}

\author{
Yuri G. Strukov and Andrew S. Belmont
}

Cold Spring Harb Protoc; doi: 10.1101/pdb.prot4903

\begin{tabular}{|c|c|}
\hline $\begin{array}{r}\text { Email Alerting } \\
\text { Service }\end{array}$ & Receive free email alerts when new articles cite this article - click here. \\
\hline $\begin{array}{l}\text { Subject } \\
\text { Categories }\end{array}$ & $\begin{array}{l}\text { Browse articles on similar topics from Cold Spring Harbor Protocols. } \\
\text { Analysis of Gene Expression in Cultured Cells (66 articles) } \\
\text { Cell Biology, general (1382 articles) } \\
\text { Cell Culture ( } 301 \text { articles) } \\
\text { Cell Imaging (525 articles) } \\
\text { DNA Delivery/Gene Transfer (344 articles) } \\
\text { DNA Delivery/Gene Transfer, general (341 articles) } \\
\text { DNA Modification/Epigenetics (42 articles) } \\
\text { Fluorescence (517 articles) } \\
\text { Fluorescence, general (341 articles) } \\
\text { Fluorescent Proteins ( } 259 \text { articles) } \\
\text { Imaging/Microscopy, general (579 articles) } \\
\text { In Vivo Imaging (334 articles) } \\
\text { In Vivo Imaging, general (168 articles) } \\
\text { Labeling for Imaging (339 articles) } \\
\text { Live Cell Imaging (274 articles) } \\
\text { Molecular Biology, general (1293 articles) } \\
\text { Non-Viral Methods (226 articles) } \\
\text { Preparation of Macromolecules and Introduction into Cells (97 articles) } \\
\text { Proteins and Proteomics, general (575 articles) } \\
\text { Tagging Proteins (57 articles) } \\
\text { Visualization (524 articles) } \\
\text { Visualization, general (369 articles) }\end{array}$ \\
\hline
\end{tabular}

